\title{
4D-STEM for Quantitative Imaging of Magnetic Materials with Enhanced Contrast and Resolution
}

Kayla X. Nguyen ${ }^{1}$, Prafull Purohit ${ }^{2}$, Robert Hovden ${ }^{1}$, Emrah Turgut $^{1}$, Mark W. Tate ${ }^{2}$, Lena F. Kourkoutis $^{1,3}$, Gregory D. Fuchs ${ }^{1,3}$, Sol M. Gruner ${ }^{2,3}$, David A. Muller ${ }^{1,3}$

${ }^{1}$ School of Applied and Engineering Physics, Cornell University, Ithaca, NY, USA

2. Department of Physics, Cornell University, Ithaca, NY, USA

${ }^{3}$ Kavli Institute at Cornell for Nanoscale Science, Ithaca, NY USA

There is a rich variety of methods for imaging magnetic fields in TEM [1], but currently far fewer options are available for the STEM geometry. Differential phase contrast (DPC) imaging with a STEM quadrant detector provides a signal approximately proportional to the deflection of the electron beam from the local magnetic field [2]. While DPC is enjoying a resurgence, there are multiple constraints to this technique including limited dynamic ranges of the detectors, nonlinearities introduced by the signal normalization when the detector is not perfectly centered, and changing beam shape. However, if a detector can determine the true center of mass (COM) of a diffraction pattern [3], and thereby is able to obtain the probability current flow, the shifts from COM can be quantitatively connected to the projected Lorentz force [4]. Here, we demonstrate a true COM imaging system to quantitatively reconstruct magnetic fields and domains, where the full convergent beam electron diffraction (CBED) pattern, containing all crystallographic information, is collected at high dynamic range, and high speed using an electron microscopy pixelated array detector (EMPAD) developed at Cornell [5]. This allows for the identification of effects arising from sample thickness, tilt, and diffraction as well as true magnetic deflections.

Detection accuracy is set by the signal to noise ratio (SNR) and detector dynamic range. The EMPAD, a detector intended for diffraction imaging (Figure 1a), has a SNR of 140:1 for a single electron and high dynamic range allowing detection of 1 to $>10^{6}$ electrons per pixel per image, at 1162 frames per second [5]. Figure $1 \mathrm{~b}$ shows an unsaturated CBED pattern of $\mathrm{SrTiO}_{3}$. Changes in the scattered beam's momentum are directly measured to reconstruct the in-plane magnetic fields showing the $\mathrm{x}$-component of the magnetic ripples from cobalt (Figure 1c) [5].

Although DPC-STEM and transport of intensity reconstructions from Lorentz-TEM are roughly connected [4], the incoherent response function for DPC-STEM effectively doubles its information limit [3], and allows all the information needed to be recorded in a single, in-focus scan. To compare the methods, we examined magnetic domains in FeGe on holey carbon at cryogenic temperatures (173K). The in-focus Lorentz TEM image (Figure 2 a) and the under-focus image (Figure 2b) show contrast both from magnetic domains and also grain structure. Our true COM imaging shows only the components related to the magnetic field, while concurrently capturing the full crystallographic information. As a starting point for ptychography and to improve the collection efficiency of BF-STEM, we cross correlate individual bright field (BF) images of cobalt regions inside the central beam (Figure 3a). We show that by resizing these $\mathrm{BF}$ images, and cross correlating them to correct for the phase shift across the lens, the image pixel size is quadrupled, and contrast and resolution are enhanced (Figure 3b).

By collecting the full CBED, the EMPAD allows for simultaneous measurement of multiple signals annular dark field (ADF), bright field (BF), to more sophisticated techniques - DPC, COM imaging, and 
ptychography, making it easier to quantify data and correct for local sample thickness and diffraction artifacts [6].

\section{References:}

[1] H. Boersch, et al., Naturwissenschaften., 46, 574 (1959).

[2] J. Chapman, J. Phys. D. 17, 623 (1984).

[3] E. M. Waddell and J. N. Chapman. Optik 54 (1979), 83-96

[4] A. Lubk and J. Zweck. Physical Review A 91 (2015), 023805

[5] M.W. Tate, et al. Microscopy and Microanalysis, First View (2016), doi:10.1017/S1431927615015664.

[6] Supported by the NSF MRSEC program (DMR 1120296) and the Kavli Institute at Cornell.

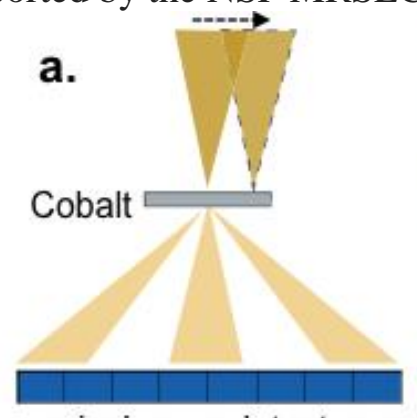

pixel array detector
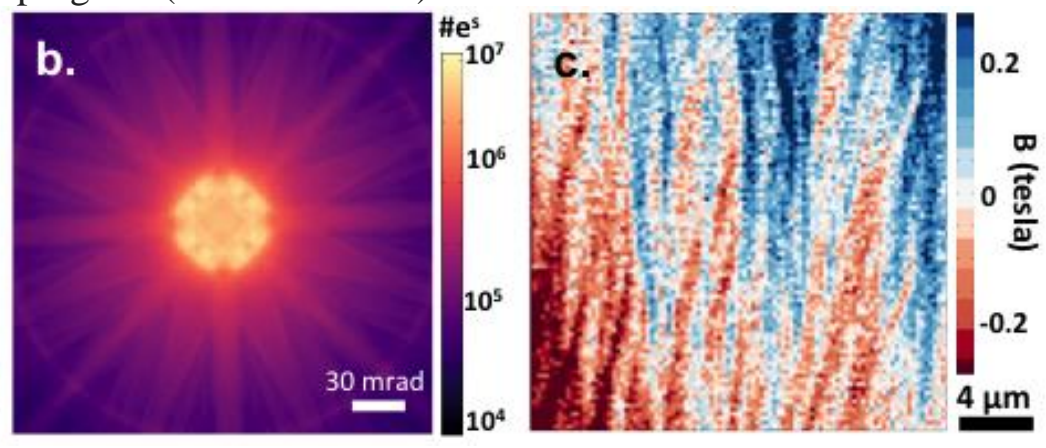

Figure 1. a) Schematic of Lorentz-STEM mode with the EMPAD. b) 10000 CBED pattern summed to show diffracted disks of $\mathrm{SrTiO}_{3}$, taken at $1 \mathrm{~ms}$ per diffraction pattern. c) Magnetic deflections from Co.
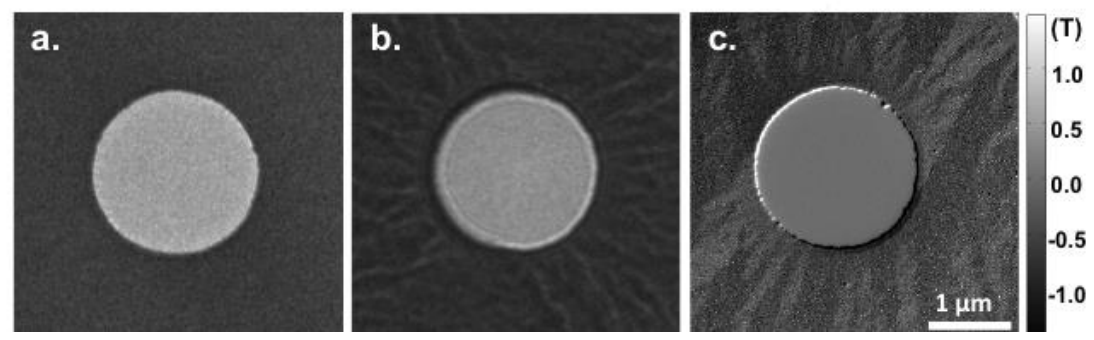

Figure 2. Lorentz TEM images of FeGe on holey carbon taken at $176 \mathrm{~K}$ a) in-focus and b) under-focus. c) Magnetic deflections from true center of mass (COM) imaging mode.
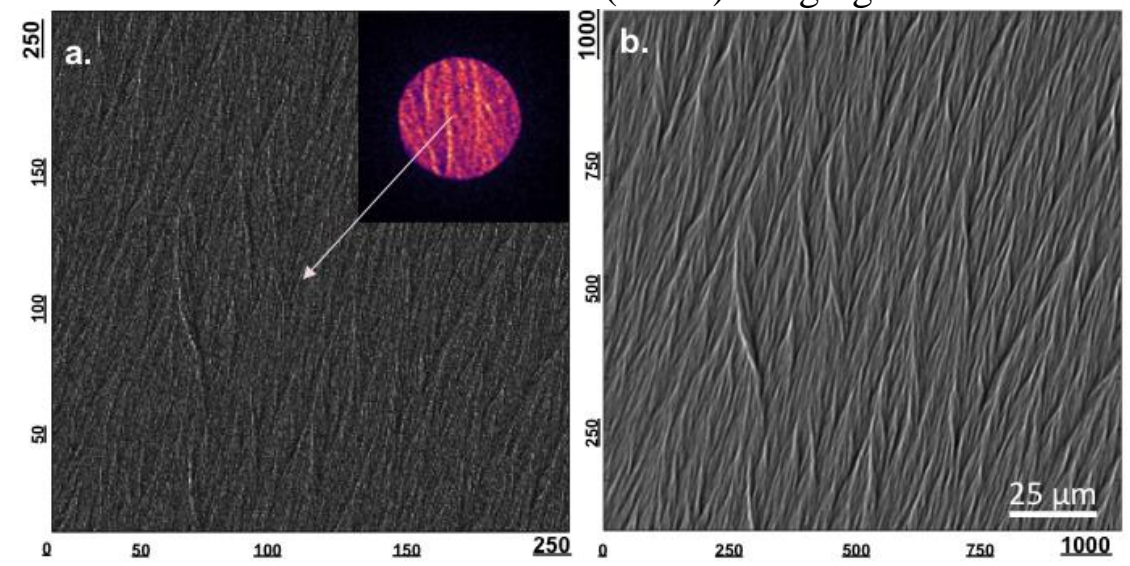

Figure 3. Dose-efficient BF-Lorentz STEM of Co. a) Bright field image taken from a single pixel in the central disk shadow image at a large defocus. b) Enhanced-resolution image of magnetic ripples in Co from the cross-correlation of different bright field images constructed from all pixels across the shadow image. (b) is both more dose-efficient - it uses all the scattered electrons, but also allows subpixel sampling from the information contained in the shadow image. 